International Journal of Pure and Applied Mathematics

Volume 105 No. 1 2015, 115-126

ISSN: 1311-8080 (printed version); ISSN: 1314-3395 (on-line version)

url: http://www.ijpam.eu

doi: http://dx.doi.org/10.12732/ijpam.v105i1.10

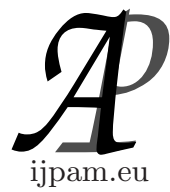

\title{
TIME EVOLUTION OF QUANTUM SYSTEMS RELATED TO THE QUANTUM ZENO SCHEME
}

\author{
Karl-Heinz Fichtner ${ }^{1}$, Kei Inoue ${ }^{2} \S$ \\ ${ }^{1}$ Friedrich-Schiller-Universität Jena \\ School of Mathematics and Computer Science \\ Institute of Applied Mathematics \\ 07737, Jena, GERMANY \\ ${ }^{2}$ Department of Electrical Engineering \\ Tokyo University of Science, Yamaguchi \\ Sanyo-Onoda, Yamaguchi, 756-0884, JAPAN
}

\begin{abstract}
At first glance it is surprising that there are papers describing experiments fitting to the quantum Zeno effect and on the other hand there are very similar experiments which seem to contradict to the quantum Zeno effect.

Now the quantum Zeno effect should be related to a suppression of the time evolution in case of an "almost continuous measurement". What does that mean? In this paper we discuss two possibilities to give a precise notion of such a suppression. That completes the considerations of the quantum Zeno scheme in our previous paper (K.-H. Fichtner and K. Inoue, 2013).
\end{abstract}

AMS Subject Classification: 81P15, 46L53, 47N30

Key Words: quantum Zeno effect, quantum measurements

\section{Introduction}

In order to avoid technical problems and complicated calculations we restrict our considerations to the case of a finite dimensional Hilbert space $H$.

Received: August 15, 2015

(c) 2015 Academic Publications, Ltd.

${ }^{\S}$ Correspondence author url: www.acadpubl.eu 
Let $\left(V_{t}:=e^{i t h}\right)_{t \geq 0}$ be a semigroup of unitary operators on $H$ representing the time evolution of a quantum system, where $h$ denotes a self-adjoint operator on $H$.

$\rho_{0}=\rho$ denotes the state of the quantum system at time $t_{0}=0$.

Furthermore we consider a sequence of time

$$
t_{k}:=\frac{k T}{n}, \quad k=0,1, \ldots, n, \quad T>0
$$

with equidistant times on the time interval $[0, T]$.

Now, we perform measurements at time $t_{k}(k=1, \ldots, n-1)$ related to the self-adjoint operator $B$ on $H$ and finally at time $t_{n}$ we perform a measurement related to the self-adjoint operator $A(\neq B)$ on $H$. That means we will consider the following scheme we call to be the quantum Zeno scheme.

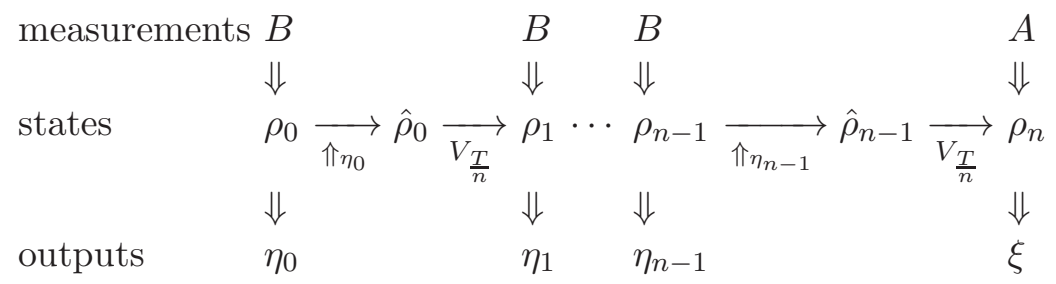

The above procedure consists of the following steps:

Step 1: Starting with the state $\rho_{0}$ one performs a measurement according to $B$ at time $t_{0}=0 . \quad \eta_{0}$ denotes the output of this measurement. Then according to von Neumann's rule the state $\rho_{0}$ changes into a certain state $\hat{\rho}_{0}$ depending on $\eta_{0}$. Finally time evolution according to $V_{\frac{T}{n}}=V_{t_{1}-t_{0}}$ transforms the state $\hat{\rho}_{0}$ into the state $\rho_{1}$ representing the state of the quantum system at time $t_{1}$.

Step 2: Starting with the state $\rho_{1}$ we repeat the procedure described above, i.e., first we perform a measurement according to $B$, and then apply the time evolution according to $V_{\frac{T}{n}}=V_{t_{2}-t_{1}}$. Thus as the result of step 2 we obtain the output of the measurement $\eta_{1}$ and a state $\rho_{2}$ at time $t_{2}$.

Repeating the procedure again and again we obtain as the result of the Step (n-1) the value $\eta_{n-1}$ being the output of the measurement according to $B$ at time $t_{n-1}$, and the state $\rho_{n}$ of the quantum system at time $t_{n}=T$ depending on $\left(\eta_{k}\right)_{k=0}^{n-1}$. 
Step n: One performs a measurement according to $A$ at time $T$ with output $\xi$.

Concerning the quantum Zeno effect, in order to make precise what suppression of time evolution could mean we will consider the following two possibilities.

Possibility 1: Let us consider measurements according to $B n$ times without any distance of time between these measurements. In terms of our scheme one can put formally $T=0$. Then there holds $\eta_{k}=\eta_{0}(k=1, \ldots, n-1)$. For that reason one could relate the quantum Zeno effect in terms of our scheme to the statement

$$
P\left(\eta_{k}=\eta_{0} ; k=1, \ldots, n-1\right) \underset{n \rightarrow+\infty}{\longrightarrow} 1 .
$$

That was already proved in [10].

Possibility 2: Neglecting the outputs $\eta_{0}, \ldots, \eta_{n-1}$ of the measurements according to $B$ our scheme leads to a transformation of the initial state $\rho$ into a state $\rho_{n, T, B, h}$ at time $T$ being the mixture of the states $\rho_{n}=\rho_{\left(\eta_{k}\right)_{k=0}^{n-1}}$ with respect to the distribution of the outputs $\left(\eta_{k}\right)_{k=0}^{n-1}$. Then one could relate the quantum Zeno effect to the statement

$$
\rho_{n, T, B, h} \underset{n \rightarrow+\infty}{\longrightarrow} \rho, \text { i.e., } \operatorname{tr}\left(A \rho_{n, T, B, h}\right) \underset{n \rightarrow+\infty}{\longrightarrow} \operatorname{tr}(A \rho) .
$$

But that does not hold in general. Of course, in the case of the trivial measurement related to the unity operator $B=1_{H}$ there is no suppression of the time evolution, i.e., we cannot expect that one can observe the quantum Zeno effect in the sense of possibility 2 in the general case of an operator $B$. Even in the case of a nontrivial measurement related to an operator $B$ having at least one eigenspace with dimension higher than 1 one can construct counter examples. For that reason in the following we will restrict our considerations to the case of an operator $B$ having only one-dimensional eigenspaces.

Before starting let us mention some motivations of our work:

There are certain quantum models of brain activities where the quantum Zeno effect plays an important role. For details we refer to [10, 17, 19].

Now, let us assume that the brain acts like a quantum system. We consider the self-adjoint operator $B$ related to EEG-measurement. Modern measuring devices are able to perform 50,000 measurements per second. Thus in the cases of our scheme we can put $T=1 \mathrm{sec}$ and $n=50,000$. Then our procedures 
could be considered as an "almost continuous measurement" according to $B$. Thus we get (cf. possibility 1)

$$
P\left(\eta_{k}=\eta_{0} ; k=1, \ldots, n-1\right) \approx 1 .
$$

That makes statistical investigations more efficient and it fits to certain experimental experiences.

If on the other hand one would have a total suppression of the time evolution of the brain in the sense of possibility 2 , then the usual activities of the brain would be stopped, i.e., the patient would die. That would contradict to the experiences. Moreover specialists are convinced that EEG-measurements do not harm the brain [21]. From the mathematical point of view the reason could be that even in the case of a discretized model of EEG-measurements the structure of the eigenspaces of the corresponding operator seems to be very complicated.

Further let us mention that essential elements of our quantum model of certain brain activities are socalled self-collapses $[2,3,4,5,6,7,8,9,11]$. Now it is known that more than $10^{11}$ self-collapses per second can occur and these self-collapses cause changes of a quantum state similar to the effect of measurements. The corresponding self-adjoint operator is a relatively simple one but there are higher dimensional eigenspaces. For that reason it would be interesting to check what kind of modifications of the quantum Zeno effect one could observe in case of higher dimensional eigenspaces. But that would be another problem.

\section{On the Quantum Zeno Effect}

As we have already mentioned before, in order to avoid technical problems and complicated calculations we restrict our considerations to the case of a finite-dimensional Hilbert space $H$ with dimension $d$. Further we consider a self-adjoint operator $B$ on $H$ having only one-dimensional eigenspaces, i.e., we have the representation

$$
B=\sum_{k} b_{k} \operatorname{Pr}_{\psi_{k}}
$$

where $\left(\psi_{k}\right)_{k=1}^{d}$ represents a CONS in $H$, and there holds $b_{k} \neq b_{j}(k \neq j)$.

Now, let $V=\left(V_{t}:=e^{i t h}\right)_{t \geq 0}$ be a semigroup of unitary operators on $H$ representing the time evolution of a quantum system, where $h$ denotes a selfadjoint operator on $H$. 
We consider the following procedure: Fixing a state $\rho$ of a quantum system at time $t_{0}=0, T>0$, and $n=2,3, \ldots$ we perform measurements according to $B$ interrupting the time evolution at times

$$
t_{k}:=\frac{k T}{n} \quad(k=1, \ldots, n-1) .
$$

That means we consider repeated measurements according to $B$ starting at time $t_{1}$ with equidistant times. Formally we identify the random outputs of these measurements with random numbers $\eta_{1}, \ldots, \eta_{n-1}$. Then using theorem 3 in [10] one easily checks

\section{Theorem 2.1. It holds}

$$
P\left(\eta_{k}=\eta_{1} ; k=1, \ldots, n-1\right) \underset{n \rightarrow+\infty}{\longrightarrow} 1 .
$$

Now, related to our scheme we complete the considered procedure adding a measurement according to a self-adjoint operator $A$ at time $T$. Then neglecting the outputs $\eta_{1}, \ldots, \eta_{n-1}$ of the measurements according to $B$ leads to a linear channel $C_{T, n}$ acting on the set $S(H)$ of self-adjoint operators on $H$, such that the expectation of the output $\xi_{n}$ of the measurement according to $A$ is given by

$$
E \xi_{n}=\operatorname{tr}\left(\rho C_{T, n}(A)\right) .
$$

Following the Section 2 in [10] a precise definition of the channel $C_{T, n}$ we obtain as follows:

First we define linear channels acting on $S(H)$ putting

$$
\begin{array}{rll}
K_{t}:=V_{t}^{*} A V_{t} \quad & (t \geq 0 ; A \in S(H)), \\
W_{j}(A):=\operatorname{Pr}_{\psi_{j}} A \operatorname{Pr}_{\psi_{j}} \quad(j=1, \ldots, d ; A \in S(H)), \\
Q_{\left(j_{s}\right)_{s=1}^{n-1}}:=W_{j_{1}} \circ K_{\frac{T}{n}} \circ W_{j_{2}} \circ K_{\frac{T}{n}} \circ \cdots \circ W_{j_{n-2}} \circ K_{\frac{T}{n}} \circ W_{j_{n-1}} \\
& & \left(n \geq 2 ; 1 \leq j_{1}, \ldots, j_{n-1} \leq d\right) .
\end{array}
$$

Then a precise definition of $C_{T, n}$ is given by

$$
C_{T, n}:=C_{V, B, T, n}:=\sum_{j_{1}, \ldots, j_{n-1}} K_{\frac{T}{n}} \circ Q_{\left(j_{s}\right)_{s=1}^{n-1}} \circ K_{\frac{T}{n}} .
$$

Now, let $\rho$ be a state on $H$. Further let $A$ be given by its unique representation

$$
A=\sum_{m} z_{m} \operatorname{Pr}_{H_{m}},
$$


i.e., $\left(H_{m}\right)_{m}$ is an orthogonal decomposition of $H$, and $z_{m} \neq z_{r}$ if $m \neq r$.

Again let $\xi_{n}$ denotes the output of a measurement according to $A$ at time $T$. Using (13) we get

$$
\begin{array}{r}
\operatorname{tr}\left(\rho K_{\frac{T}{n}} \circ Q_{\left(j_{s}\right)_{s=1}^{n-1}} \circ K_{\frac{T}{n}}\left(\operatorname{Pr}_{H_{m}}\right)\right)=P\left(\xi_{n}=z_{m}, \eta_{1}=j_{1}, \ldots, \eta_{n-1}=j_{n-1}\right) \\
\left(n \geq 2 ; 1 \leq j_{1}, \ldots, j_{n-1} \leq d ; m\right)(14)
\end{array}
$$

Then using (12), (13), and (14) we obtain

$$
\begin{aligned}
\operatorname{tr}\left(\rho C_{T, n}(A)\right) & =\sum_{m} z_{m} \sum_{j_{1}, \ldots, j_{n-1}} \operatorname{tr}\left(\rho K_{\frac{T}{n}} \circ Q_{\left(j_{s}\right)_{s=1}^{n-1}} \circ K_{\frac{T}{n}}\left(\operatorname{Pr}_{H_{m}}\right)\right) \\
& =\sum_{m} z_{m} \sum_{j_{1}, \ldots, j_{n-1}} P\left(\xi_{n}=z_{m}, \eta_{1}=j_{1}, \ldots, \eta_{n-1}=j_{n-1}\right) \\
& =E \xi_{n}
\end{aligned}
$$

Thus the definition of (12) of the channel $C_{T, n}$ fits to (8).

Theorem 2.2. For each state $\rho$ there holds

$$
\operatorname{tr}\left(\rho C_{T, n}(A)\right) \underset{n \rightarrow+\infty}{\longrightarrow} \operatorname{tr}\left(\rho\left(\sum_{j} W_{j}(A)\right)\right) \quad(A \in S(H)) .
$$

Remark 2.3. In the sense of our procedure the channel

$$
\hat{C}_{T, n}:=\left(\sum_{j} W_{j}{ }^{(n-1) \circ}\right.
$$

is related to the trivial time evolution according to

$$
V_{t}:=e^{i t_{0}}=1_{H} \quad(t \geq 0),
$$

i.e., there are only repeated measurements according to $B$.

Because of

$$
\left(\sum_{j} W_{j}^{(n-1) \circ}=\sum_{j} W_{j}\right.
$$

theorem 2.2 implies that

$$
\operatorname{tr}\left(\rho C_{T, n}(A)\right)-\operatorname{tr}\left(\rho \hat{C}_{T, n}(A)\right) \underset{n \rightarrow+\infty}{\longrightarrow} 0 \quad(A \in S(H)) .
$$


That makes precise what suppression of time evolution in the sense of possibility 2 could mean.

Remark 2.4. Let $Z_{T, n}(\rho)$ be the state characterized by

$$
\operatorname{tr}\left(A Z_{T, n}(\rho)\right)=\operatorname{tr}\left(\rho C_{T, n}(A)\right) \quad(A \in S(H))
$$

Then $Z_{T, n}(\rho)$ represents the state at time $T$ in the sense of our scheme. Further, measurement according to $B$ neglecting the output transforms the state $\rho$ into the state $W_{B}(\rho)$ characterized by

$$
\operatorname{tr}\left(W_{B}(\rho) A\right)=\operatorname{tr}\left(\rho \sum_{j} W_{j}(A)\right) \quad(A \in S(H)) .
$$

Then theorem 2.2 may be reformulated as follows

$$
Z_{T, n}(\rho) \underset{n \rightarrow+\infty}{\longrightarrow} W_{B}(\rho) .
$$

For that reason we obtain

$$
Z_{T, n}(\rho) \underset{n \rightarrow+\infty}{\longrightarrow} \rho
$$

if $W_{B}(\rho)=\rho$. For instance that holds if $\rho$ is an eigenstate of the operator $B$ (cf. example A. Turing - already discussed in [10]).

\section{Proof of Theorem 2.2}

The following lemma represents an essential part of the proof of the theorem.

Lemma 3.1. Let be $t>0, j=1, \ldots, d$. Then it holds

$$
\left(W_{j}\left(V_{\frac{t}{n}}\right)\right)^{n}=\left(\operatorname{Pr}_{\psi_{j}} V_{\frac{t}{n}} \operatorname{Pr}_{\psi_{j}}\right)^{n} \underset{n \rightarrow+\infty}{\longrightarrow} e^{i t\left\langle\psi_{j} \mid h \psi_{j}\right\rangle} \operatorname{Pr}_{\psi_{j}} .
$$

Proof. We use the following representation of $h$

$$
h=\sum_{k} a_{k} \operatorname{Pr}_{\varphi_{k}}
$$

where $\left(\varphi_{k}\right)_{k=1}^{d}$ is a CONS. 
Then we get

$$
\operatorname{Pr}_{\psi_{j}} V_{\frac{t}{n}} \operatorname{Pr}_{\psi_{j}}=\sum_{k} e^{i \frac{t}{n} a_{k}} \operatorname{Pr}_{\psi_{j}} \operatorname{Pr}_{\varphi_{k}} \operatorname{Pr}_{\psi_{j}} \quad(k, j=1, \ldots, d) .
$$

Simple calculations show

$$
\operatorname{Pr}_{\psi_{j}} \operatorname{Pr}_{\varphi_{k}} \operatorname{Pr}_{\psi_{j}} \psi=\left|\left\langle\varphi_{k} \mid \psi_{j}\right\rangle\right|^{2} \operatorname{Pr}_{\psi_{j}} \psi \quad(\psi \in H),
$$

i.e., we have

$$
\operatorname{Pr}_{\psi_{j}} \operatorname{Pr}_{\varphi_{k}} \operatorname{Pr}_{\psi_{j}}=\left|\left\langle\varphi_{k} \mid \psi_{j}\right\rangle\right|^{2} \operatorname{Pr}_{\psi_{j}} \quad(k, j=1, \ldots, d) .
$$

Using (29) we get

$$
\left\langle\psi_{j} \mid \operatorname{Pr}_{\psi_{j}} \operatorname{Pr}_{\varphi_{k}} \operatorname{Pr}_{\psi_{j}} \psi_{j}\right\rangle=\left|\left\langle\varphi_{k} \mid \psi_{j}\right\rangle\right|^{2} \quad(k, j=1, \ldots, d) .
$$

Because of $1_{H}=\sum_{k} \operatorname{Pr}_{\varphi_{k}}$ that implies

$$
\sum_{k}\left|\left\langle\varphi_{k} \mid \psi_{j}\right\rangle\right|^{2}=1 \quad(j=1, \ldots, d)
$$

Furthermore, using (26), and (30) we obtain

$$
\left\langle\psi_{j} \mid h \psi_{j}\right\rangle=\sum_{k} a_{k}\left|\left\langle\varphi_{k} \mid \psi_{j}\right\rangle\right|^{2} \quad(j=1, \ldots, d) .
$$

Finally, using (27), and (29) we obtain

$$
\left(\operatorname{Pr}_{\psi_{j}} V_{\frac{t}{n}} \operatorname{Pr}_{\psi_{j}}\right)^{n}=\left(\sum_{k} e^{i \frac{t}{n} a_{k}}\left|\left\langle\varphi_{k} \mid \psi_{j}\right\rangle\right|^{2}\right)^{n} \operatorname{Pr}_{\psi_{j}}
$$

Now we put

$$
\alpha_{n}:=n \sum_{k}\left(e^{i \frac{t}{n} a_{k}}-1\right)\left|\left\langle\varphi_{k} \mid \psi_{j}\right\rangle\right|^{2} .
$$

Because of (31), and (34) we get

$$
\left(\sum_{k} e^{i \frac{t}{n} a_{k}}\left|\left\langle\varphi_{k} \mid \psi_{j}\right\rangle\right|^{2}\right)^{n}=\left(1+\frac{\alpha_{n}}{n}\right)^{n}
$$

One easily checks

$$
\alpha_{n} \underset{n \rightarrow+\infty}{\longrightarrow} i t \sum_{k} a_{k}\left|\left\langle\varphi_{k} \mid \psi_{j}\right\rangle\right|^{2}
$$


Thus using (36), (35), (33), and (32) we can conclude that there holds

$$
\left(\operatorname{Pr}_{\psi_{j}} V_{\frac{t}{n}} \operatorname{Pr}_{\psi_{j}}\right)^{n} \underset{n \rightarrow+\infty}{\longrightarrow} e^{i t\left\langle\psi_{j} \mid h \psi_{j}\right\rangle} \operatorname{Pr}_{\psi_{j}}
$$

Because of the definition of $W_{j}$ we obtain immediately

$$
\left(W_{j}\left(V_{\frac{t}{n}}\right)\right)^{n}=\left(\operatorname{Pr}_{\psi_{j}} V_{\frac{t}{n}} \operatorname{Pr}_{\psi_{j}}\right)^{n} .
$$

That completes the proof of the lemma.

Remark 3.2. From lemma 3.1 we immediately obtain that there holds

$$
\sum_{j}\left(W_{j}\left(V_{\frac{t}{n}}\right)\right)^{n} \underset{n \rightarrow+\infty}{\longrightarrow} \hat{V}_{t}
$$

where

$$
\hat{V}_{t}:=e^{i t \hat{h}}
$$

with the self-adjoint operator $\hat{h}$ defined by

$$
\hat{h}:=\sum_{k}\left\langle\psi_{k} \mid h \psi_{k}\right\rangle \operatorname{Pr}_{\psi_{k}} .
$$

\section{The proof of theorem 2.2:}

Using theorem 2.1 we obtain

$$
\sum_{\exists(k, s) j_{k} \neq j_{s}} P\left(\xi=z_{m}, \eta_{1}=j_{1}, \ldots, \eta_{n-1}=j_{n-1}\right) \underset{n \rightarrow+\infty}{\longrightarrow} 0 .
$$

Because of (14) that implies

$$
\sum_{\exists(k, s) j_{k} \neq j_{s}} \operatorname{tr}\left(\rho K_{\frac{T}{n}} \circ Q_{\left(j_{s}\right)_{s=1}^{n-1}} \circ K_{\frac{T}{n}}\left(\operatorname{Pr}_{H_{m}}\right)\right) \underset{n \rightarrow+\infty}{\longrightarrow} 0 .
$$

Further, one easily checks that it holds

$$
\begin{aligned}
Q_{(j)_{s=1}^{n-1}}(D)= & \left(\left(\operatorname{Pr}_{\psi_{j}} V_{\frac{T}{n}} \operatorname{Pr}_{\psi_{j}}\right)^{n-1}\right)^{*} W_{j}(D)\left(\operatorname{Pr}_{\psi_{j}} V_{\frac{T}{n}} \operatorname{Pr}_{\psi_{j}}\right)^{n-1} \\
& (D \in S(H) ; j=1, \ldots, d)) .
\end{aligned}
$$


Using (44), lemma 3.1 , and $K_{\frac{T}{n}} \underset{n \rightarrow+\infty}{\longrightarrow} 1_{S(H)}$ we obtain

$$
\operatorname{tr}\left(\rho K_{\frac{T}{n}} \circ Q_{(j)_{s=1}^{n-1}} \circ K_{\frac{T}{n}}\left(\operatorname{Pr}_{H_{m}}\right)\right) \underset{n \rightarrow+\infty}{\longrightarrow} \operatorname{tr}\left(\rho W_{j}\left(\operatorname{Pr}_{H_{m}}\right)\right) .
$$

Now, because of (15), (43), and (45) we can conclude

$$
\operatorname{tr}\left(\rho C_{T, n}(A)\right) \underset{n \rightarrow+\infty}{\longrightarrow} \operatorname{tr}\left(\rho\left(\sum_{j} W_{j}(A)\right)\right) .
$$

That completes the proof of theorem 2.2.

Remark 3.3. The proof of the theorem 2.2 shows that our procedure is related to the unitary channels $C_{t, \infty}$ on $S(H)$ defined by

$$
C_{t, \infty}:=e^{-i t \hat{h}} A e^{i t \hat{h}} \quad(t \geq 0, A \in S(H)) .
$$

One easily checks that there holds

$$
\sum_{j} W_{j} \circ C_{t, \infty}=\sum_{j} W_{j}
$$

Thus we can conclude that $W_{B}(\rho)$ is invariant with respect to the time evolution according to $\hat{V}=\left(\hat{V}_{t}\right)$, i.e., our procedure (cf. theorem 2.2, remark 2.4) leads to a mapping $\rho \rightarrow W_{B}(\rho)$ from the set of states into the set of $\hat{V}$-invariant states.

\section{References}

[1] A. Degasperis, L. Fonda and G.C. Ghirardi, Does the lifetime of an unstable system depend on the measuring apparatus?, Il Nuovo Cimento, 21 A, No.3 (1974), 471-484.

[2] K.-H. Fichtner and L. Fichtner, Bosons and a quantum model of the brain, FSU Jena, Faculty of Mathematics and Informatics, Jena, Jenaer Schriften zur Mathematik und Informatik, Math/Inf/08/05 (2005), 27 pages.

[3] K.-H. Fichtner and L. Fichtner, Quantum models of brain activities I Recognition of signals, In J.C. Garcia, R. Quezada, and S.B. Sontz, editors, Quantum Probability and Related topics, QP-PQ: Quantum Probability and White Noise Analysis, XXIII (2008), 135-144. 
[4] K.-H. Fichtner, L. Fichtner, W. Freudenberg, and M. Ohya, On a mathematical model of brain activities, In Quantum Theory, Reconsideration of Foundations-4, AIP Conference Proceedings, 962 (2007), 85-90.

[5] K.-H. Fichtner, L. Fichtner, W. Freudenberg, and M. Ohya, On a quantum model of the recognition process, In L. Accardi, W. Freudenberg, and M. Ohya, editors, Quantum Bio-Informatics, QP-PQ: Quantum Probability and White Noise Analysis, XXI (2008), 64-84, .

[6] K.-H. Fichtner, L. Fichtner, W. Freudenberg, and M. Ohya, On a quantum model of the brain activities, In L. Accardi, W. Freudenberg, and M. Ohya, editors, Quantum Bio-Informatics III, QP-PQ:Quantum Probability and White Noise Analysis, XXVI (2010), 81-92.

[7] K.-H. Fichtner, L. Fichtner, W. Freudenberg, and M. Ohya, Quantum models of the recognition process - on a convergence theorem, Open Systems and Information Dynamics, 17, No.2 (2010), 161-187.

[8] K.-H. Fichtner, L. Fichtner, W. Freudenberg, and M. Ohya, Self-collapses of quantum systems and brain activities, In L. Accardi, W. Freudenberg, and M. Ohya, editors, Quantum Bio-Informatics IV, QP-PQ: Quantum Probability and White Noise Analysis, XXVIII (2011), 101-115.

[9] K.-H. Fichtner, L. Fichtner, K. Inoue, and M. Ohya, Internal noise caused by the memory, Open Systems and Information Dynamics, 18, No.4 (2011), 405-422.

[10] K.-H. Fichtner and K. Inoue, On the quantum Zeno effect and time series related to quantum measurements, Applied Mathematics, 4, No.10C (2013), 61-69.

[11] K.-H. Fichtner, K. Inoue, and M. Ohya, On a quantum model of brain activities - distribution of the outcomes of EEG-measurements and random point fields, Open Systems and Information Dynamics, 19, No.4 (2012), 1250025 .

[12] P. Facchi, D.A. Lidar and S. Pascazio, Unification of dynamical decoupling and the quantum Zeno effect, Phys. Rev. A, 69 (2004), 032314.

[13] R. Hari, O.V. Lounasmaa, Neuromagnetism: tracking the dynamics of the brain, Physics World, 13 (2000), 33-38. 
[14] S. Hameroff and R. Penrose, Orchestrated objective reduction of quantum coherence in brain microtubules: the "Orch OR" model for consciousness, Mathematics and Computer Simulation, 40 (1996), 453-480.

[15] J. von Neumann, Mathematische Grundlagen der Quantenmechanik, Springer, Germany (1932).

[16] T. Nakanishi, K. Yamane, and M. Kitano, Absorption-free optical control of spin systems: the quantum Zeno effect in optical pumping, Phys. Rev. A, 65 (2001), 013404.

[17] R. Penrose, The Emperor's New Mind: Concerning Computers, Minds and The Laws of Physics, Oxford University Press, UK (1989).

[18] B. Misra and E.C.G. Sudarshan, The Zeno's paradox in quantum theory, J. Math. Phys., 18, No.4 (1977), 756-763.

[19] J.M. Schwartz, H.P. Stapp and M. Beauregard, Quantum physics in neuroscience and psychology: a neurophysical model of mind-brain interaction, Philosophical Transactions of the Royal Society of London B, 360, No.1458 (2005), 1309-1327.

[20] C. Teuscher and D. Hofstadter, Alan Turing: Life and Legacy of a Great Thinker, Springer-Verlag, Germany (2004).

[21] W. Tirsch, Biomedizinische Relevanz der quantitativen EEG Analyse, Shaker Verlag, Germany (2009). 